\title{
Brane universes tested by supernovae
}

\author{
Mariusz P. Dąbrowski \\ Institute of Physics, University of Szczecin, Wielkopolska 15, 70-451 Szczecin, Poland \\ Włodzimierz Godłowskif and Marek Szydłowskif \\ Astronomical Observatory, Jagiellonian University, 30-244 Krakow, ul. Orla 171, Poland
}

(Dated: November 10, 2018)

\begin{abstract}
We discuss observational constrains coming from supernovae Ia [3] imposed on the behaviour of the Randall-Sundrum models. In the case of dust matter on the brane, the difference between the best-fit general relativistic model with a $\Lambda$-term [3] and the best-fit brane models becomes detectable for redshifts $z>0.6$. It is interesting that brane models predict brighter galaxies for such redshifts which is in agreement with the measurement of the $z=1.7$ supernova [6] and with the New Data from the High Z Supernovae Search Team [7. We also demonstrate that the fit to supernovae data can also be obtained, if we admit the "super-negative" dark energy $p=-(4 / 3) \varrho$ on the brane, where the dark energy in a way mimics the influence of the cosmological constant. It also appears that the dark energy enlarges the age of the universe which is demanded in cosmology. Finally, we propose to check for dark radiation and brane tension by the application of the angular diameter of galaxies minimum value test.
\end{abstract}

PACS numbers: 04.20.Jb,04.65.+e,98.80.Hw

The idea of brane universes has originated from Hořava and Witten [1] followed by Randall and Sundrum [2]. Brane models admit new parameters which are not present in standard cosmology (brane tension $\lambda$ and dark radiation $\mathcal{U})$. From the astronomical observations of supernovae Ia [3, 4] one knows that the universe is now accelerating and the best-fit model is for the 4-dimensional cosmological constant density parameter $\Omega_{\Lambda_{(4)}, 0}=0.72$ and for the dust density parameter $\Omega_{m, 0}=0.28$ (index "0" refers to the present moment of time). In other words, only the exotic (negative pressure) matter in standard cosmology can lead to this global effect. On the other hand, in brane models the $\varrho^{2}$ quadratic contribution in the energy density even for a small negative pressure, contributes effectively as the positive pressure, and makes brane models less accelerating. In this paper we argue that in order to avoid this problem one requires much stronger negative pressure $p<-\varrho$ matter to be present on the brane (cf. Ref. [5]) unless the new HZT data shows the $z>1$ supernovae are brighter than expected [6, 7].

In Ref. 8] we gave the formalism to express dynamical equations in terms of dimensionless observational density parameters $\Omega$. Following Refs. [9, 10, 11] we introduce the notation useful for this purpose. In this notation the Friedmann equation for brane universes takes the form

$$
\frac{1}{a^{2}}\left(\frac{d a}{d t}\right)^{2}=\frac{C_{G R}}{a^{3 \gamma}}+\frac{C_{\lambda}}{a^{6 \gamma}}-\frac{k}{a^{2}}+\frac{\Lambda_{(4)}}{3}+\frac{C_{\mathcal{U}}}{a^{4}},
$$

where $a(t)$ is the scale factor, $k=0, \pm 1$ the curvature index, $\Lambda_{(4)}$ the 4-dimensional cosmological contant, and $\gamma$ the barotropic index $(p=(\gamma-1) \varrho, p$ - the pressure, $\varrho$ - the energy density). In Eq. (1) we have defined the appropriate constants $\left(\kappa_{(4)}^{2}=8 \pi G\right) C_{G R}=(1 / 3) \kappa_{(4)}^{2} a^{3 \gamma} \varrho$, $C_{\lambda}=1 / 6 \lambda \cdot \kappa_{(4)}^{2} a^{6 \gamma} \varrho^{2}, C_{\mathcal{U}}=2 / \kappa_{(4)}^{2} \lambda \cdot a^{4} \mathcal{U}$, and $C_{G R}$ is a of general relativistic nature, $C_{\lambda}$ comes as contribution from brane tension $\lambda$, and $C_{\mathcal{U}}$ as a contribution from dark radiation. Though in Refs. 8, 12 the Eq. (11) was studied using qualitative methods we just mention here that the cases $\gamma=0$ (cosmological constant), $\gamma=1 / 3$ (domain walls) and $\gamma=2 / 3$ (cosmic strings) can be exactly integrable in terms of elementary [16] or elliptic [9] functions. For other values of $\gamma=4 / 3 ; 1 ; 2$, the terms of the type $1 / a^{8}$ and $1 / a^{12}$ appear, and the integration involves hyperelliptic functions. In particular, oscillating non-singular solutions appear for dark energy $\gamma=-1 / 3$ |16.

In order to study observational tests we now define dimensionless observational density parameters [10, 11]

$$
\begin{aligned}
\Omega_{G R} & =\frac{\kappa_{(4)}^{2}}{3 H^{2}} \varrho, \quad \Omega_{\lambda}=\frac{\kappa_{(4)}^{2}}{6 H^{2} \lambda} \varrho^{2}, \quad \Omega_{\mathcal{U}}=\frac{2}{\kappa_{(4)}^{2} H^{2} \lambda} \mathcal{U}, \\
\Omega_{k} & =-\frac{k}{H^{2} a^{2}}, \quad \Omega_{\Lambda_{(4)}}=\frac{\Lambda_{(4)}}{3 H^{2}},
\end{aligned}
$$

where the Hubble parameter $H=\dot{a} / a$, and the deceleration parameter $q=-\ddot{a} a / \dot{a}^{2}$, so that the Friedmann equation (1) can be written down in the form

$$
\Omega_{G R}+\Omega_{\lambda}+\Omega_{k}+\Omega_{\Lambda_{(4)}}+\Omega_{\mathcal{U}}=1
$$

Note that $\Omega_{\mathcal{U}}$ in (2), despite standard radiation term, can either be positive or negative. Using (2), the equation (11) can now be rewritten as (compare Eq.(10) of [10])

$$
\Omega_{\Lambda_{(4)}}=\frac{3 \gamma-2}{2} \Omega_{G R}+(3 \gamma-1) \Omega_{\lambda}+\Omega_{\mathcal{U}}-q .
$$

It is also useful to express the curvature of spatial sections by observational parameters by using (3) and (4)

$$
-\Omega_{k}=\frac{3 \gamma}{2} \Omega_{G R}+3 \gamma \Omega_{\lambda}+2 \Omega_{\mathcal{U}}-q-1 .
$$


These relations (4) and (5) allow to write down an explicit redshift-magnitude relation (a generalized Hubble law) for the brane models to study their compatibility with astronomical data which is the subject of the present paper. Obviously, the luminosity of galaxies depends on the present densities of the different components of matter content $\Omega$ given by (2) and their equations of state reflected by the value of the barotropic index $\gamma$.

Let us consider an observer located at $r=0$ at the moment $t=t_{0}$ which receives a light ray emitted at $t=t_{1}$ from the source of the absolute luminosity $L$ located at the radial distance $r_{1}$. The redshift $\mathrm{z}$ of the source is related to the scale factor $a(t)$ at the two moments of evolution by $1+z=a\left(t_{0}\right) / a\left(t_{1}\right) \equiv a_{0} / a$. If the apparent luminosity of the source as measured by the observer is $l$, then the luminosity distance $d_{L}$ of the source is defined by the relation

$$
l=\frac{L}{4 \pi d_{L}^{2}}
$$

where

$$
d_{L}=(1+z) a_{0} r_{1} \equiv \frac{\mathcal{D}_{L}(z)}{H_{0}}
$$

and $\mathcal{D}_{L}$ is the dimensionless luminosity distance. The observed and absolute luminosities are defined in terms of K-corrected apparent and absolute magnitudes $m$ and $M$. When written in terms of $m$ and $M$, Eq.(6) yields

$$
m(z)=\mathcal{M}+5 \log _{10}\left[\mathcal{D}_{L}(z)\right]
$$

where $\mathcal{M}=M-5 \log _{10} H_{0}+25$. For homogeneous and isotropic Friedmann models one gets [11]

$$
\mathcal{D}_{L}(z)=\frac{(1+z)}{\sqrt{\mathcal{K}}} S(\chi)
$$

where $S(\chi)=\sin \chi$ for $\mathcal{K}=-\Omega_{k, 0} ; S(\chi)=\chi$ for $\mathcal{K}=1$; $S(\chi)=\sinh$ for $\mathcal{K}=\Omega_{k, 0}$. From the Friedmann equation (11) and the form of the FRW metric we have

$$
\begin{aligned}
& \chi(z)=\frac{1}{a_{0} H_{0}} \int_{0}^{z}\left\{\Omega_{\lambda, 0}\left(1+z^{\prime}\right)^{6 \gamma}+\Omega_{G R, 0}\left(1+z^{\prime}\right)^{3 \gamma}\right. \\
& \left.+\Omega_{k, 0}\left(1+z^{\prime}\right)^{2}+\Omega_{\mathcal{U}, 0}\left(1+z^{\prime}\right)^{4}+\Omega_{\Lambda_{(4), 0}}\right\}^{-1 / 2} d z^{\prime}(10)
\end{aligned}
$$

Firstly, we will study the case $\gamma=1$ (dust on the brane; we will label $\Omega_{G R}$ by $\Omega_{m}$ ). The case $\gamma=2 / 3$ (cosmic strings on the brane) has recently been studied in 15 where, in fact, $\Omega_{\mathcal{U}}$ and $\Omega_{\lambda}$ were neglected and where the term $\Omega_{m, 0}\left(1+z^{\prime}\right)^{3}$ was introduced in order to admit dust matter on the brane. This case was already presented in a different framework in Ref. 11. Secondly, we will study the case $\gamma=-1 / 3$ (dark energy on the brane [5] - we will label this type of matter with $\Omega_{d}$ instead of $\Omega_{G R}$ ).
Now we test brane models using the sample of Ref. [3]. In order to avoid any possible selection effects, we use the full sample (usually, one excludes two data points as outliers and another two points, presumably reddened, from the full sample of 60 supernovae). It means that our basic sample is the sample A of Ref. [3]. We test our model using the likelihood method [4].

First of all, we estimated the value of $\mathcal{M}$ from the sample of 18 low redshift supernovae, also testing our result by the full sample of 60 supernovae taking $\Omega_{\lambda}=$ 0 . We obtained $\mathcal{M}=-3.39$ which is in a very good agreement with the results of Refs. 13, 14. Also, we obtained for the model of Ref. [3] same value of $\chi^{2}=$ 96.5 .

Neglecting dark radiation $\Omega_{\mathcal{U}, 0}=0$ we formally got the best fit $\left(\chi^{2}=94.6\right)$ for $\Omega_{k, 0}=-0.9, \Omega_{m, 0}=0.59$, $\Omega_{\lambda, 0}=0.04, \Omega_{\Lambda, 0}=1.27$, which is completely unrealistic, because $\Omega_{m, 0}=0.59$ is too large in comparison with the observational limit (also $\Omega_{k, 0}=-0.9$ is not very realistic from the observational point of view).

However, we should note that, in fact, we have an ellipsoid of admissible models in a $3 \mathrm{D}$ parameter space $\Omega_{m, 0}$, $\Omega_{\lambda, 0}, \Omega_{\Lambda_{(4)}, 0}$ at hand. Then, we have more freedom than in the case of analysis of Ref. [3] where they had only an ellipse in a $2 \mathrm{D}$ parameter space $\Omega_{m, 0}, \Omega_{\Lambda_{(4)}, 0}$. For a flat model $\Omega_{k, 0}=0$ we obtain "corridors" of possible models (1). Formally, the best-fit flat model is $\Omega_{m, 0}=0.01$, $\Omega_{\lambda, 0}=0.09 \chi^{2}=94.7$ which is again unrealistic. In the realistic case we obtain for a flat model $\Omega_{m, 0}=0.25$, $\Omega_{\lambda, 0}=0.02, \Omega_{\Lambda_{(4)}, 0}=0.73$ with $\chi^{2}=95.6$. One should note that all realistic brane models require also the presence of the positive 4-dimensional cosmological constant $\left(\Omega_{\Lambda_{(4)}, 0} \sim 0.7\right)$.

There is a question if we could fit a model with negative $\Omega_{\lambda, 0}$ ? For instance, in a flat Universe we could fit the model with $\Omega_{m, 0}=0.35$ (too much in comparison with the observational limits on the mass of the cluster of galaxies) $\Omega_{\lambda, 0}=-0.01, \Omega_{\Lambda, 0}=0.66\left(\chi^{2}=96.3\right)$. However, it is not possible to fit any models with more negative values of $\Omega_{\lambda, 0}$, regardless the Universe is flat or not.

In Fig.1 we present plots of redshift-magnitude relations against the supernovae data. One can observe that in both cases (best-fit and best-fit flat models) the difference between brane models and a pure flat model with $\Omega_{\Lambda_{(4)}, 0}=0$ is largest for $0.6<z<0.7$ while it significantly decreases for the higher redshifts. It gives us a possibility to discriminate between general relativistic models and brane models when the data from highredshift supernovae $z>1$ is available. On the other hand, the difference between the best-fit general relativistic model with a $\Lambda$-term [3] and the best-fit brane models becomes detectable for redshifts $z>0.6$. It is interesting that brane models predict brighter galaxies for such redshifts which is in agreement with the measurement of the $z=1.7$ supernova [6] and with the New Data from 


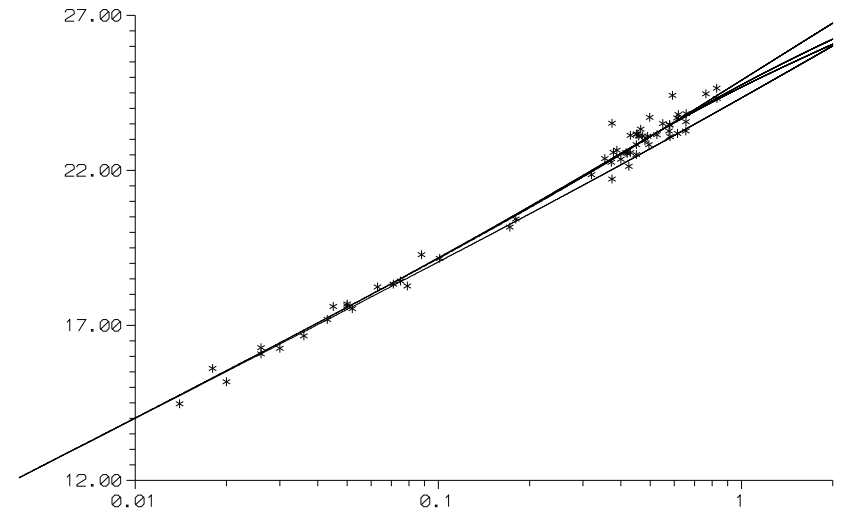

FIG. 1: Redshift-magnitude relations for $\gamma=1$ brane universes (dust on the brane). The top line is the best-fit flat model of Ref. [3] with $\Omega_{m, 0}=0.28, \Omega_{\Lambda_{(4)}, 0}=0.72$. The bottom line is a pure flat model with $\Omega_{\Lambda_{(4)}, 0}=0$. Between these two there are brane models with $\Omega_{\lambda, 0} \neq 0$ : lower - the best-fit model; higher - the best-fit flat model.

the High Z Supernovae Search Team [7]. In other words, if the farthest $z>1$ supernovae were brighter, the brane universes would be the reality.

One should note that we made our analysis without excluding any supernovae from the sample. However, from the formal point of view, when we analyze the full sample A, all models should be rejected even on the confidence level of 0.99 . One of the reason could be the fact that the assumed error bars are too small. However, in majority of papers another solution is suggested. Usually, one excludes 2 supernovae as outliers, and 2 as reddened from the sample of 42 high-redshift supernovae and eventually 2 outliers from the sample of 18 low-redshift supernovae. We decided to use the full sample A as our basic sample because a rejection of any supernovae from the sample can be the source of lack of control for selection effects. However, for completness, we also made our analysis using samples B and C. It emerged that it does not significantly changes our results, though, increases quality of the fit. Formally, the best fit for the sample B is (56 supernovae) $\left(\chi^{2}=57.3\right): \Omega_{k, 0}=-0.1 \Omega_{m, 0}=0.17$, $\Omega_{\lambda, 0}=0.06, \Omega_{\Lambda_{(4)}, 0}=0.87$. For the flat model we obtain $\left(\chi^{2}=57.3\right): \Omega_{m, 0}=0.12, \Omega_{\lambda, 0}=0.06, \Omega_{\Lambda_{(4)}, 0}=0.82$, while for a "realistic" model $\left(\Omega_{m, 0}=0.25, \Omega_{\lambda, 0}=0.02\right)$ $\chi^{2}=57.6$. Formally, the best fit for the sample C (54 supernovae) $\left(\chi^{2}=53.5\right)$ gives $\Omega_{k, 0}=0$ (flat) $\Omega_{m, 0}=0.21$, $\Omega_{\lambda, 0}=0.04, \Omega_{\Lambda_{(4)}, 0}=0.75$, while for "realistic" model $\left(\Omega_{m, 0}=0.27, \Omega_{\lambda, 0}=0.02\right) \chi^{2}=53.6$.

One should note that we have also separately estimated the value of $\mathcal{M}$ for the sample $\mathrm{B}$ and $\mathrm{C}$. We obtained $\mathcal{M}=-3.42$ which is again in a good agreement with the results of Ref. [13] (for a "combined" sample one obtains $\mathcal{M}=-3.45)$. However, if we use this value in our analysis it does not change significantly the results $\left(\chi^{2}\right.$ does not change more than 1 which is a marginal effect for $\chi^{2}$ distribution for 53 or 55 degrees of freedom).

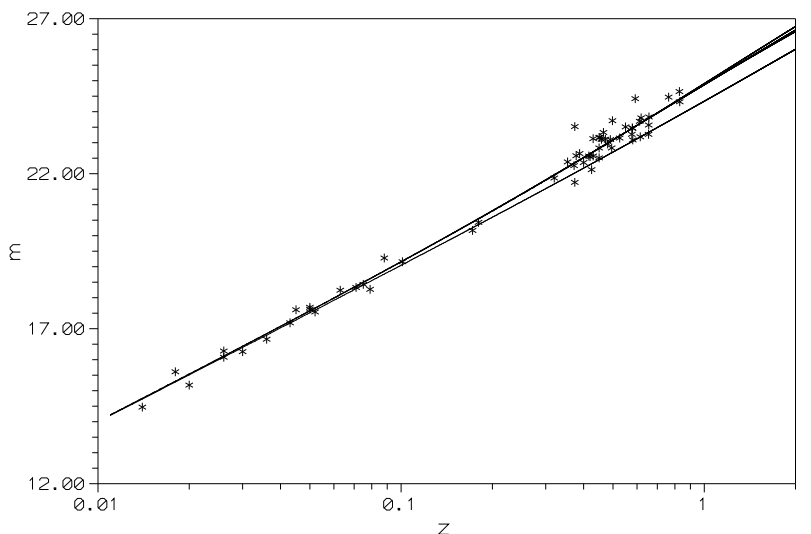

FIG. 2: A redshift-magnitude relation for $\gamma=-1 / 3$ brane universes (dark energy on the brane). The top and bottom lines are same as in Fig. 1. The brane dark energy models plots are very close to the top line of Ref. [3].

In Fig. 2 we present a redshift-magnitude relation (10) for brane models with dark energy $(\gamma=-1 / 3)$. Note that the theoretical curves are very close to that of [3] which means that the dark energy cancels the positivepressure influence of the $\varrho^{2}$ term and can simulate the negative-pressure influence of the cosmological constant to cause cosmic acceleration. From the formal point of view the best fit is $\left(\chi^{2}=95.4\right)$ for $\Omega_{k, 0}=0.2, \Omega_{d, 0}=$ $0.7, \Omega_{\lambda, 0}=-0.1, \Omega_{\mathcal{U}}=0.2, \Omega_{\Lambda_{(4)}, 0}=0$ which means that the cosmological constant must necessarily vanish. From this result we can conclude that the dark energy $p=-(4 / 3) \varrho$ can mimic the contribution from the $\Lambda_{(4)^{-}}$ term in standard models.

For the best-fit flat model $\left(\Omega_{k, 0}=0\right)$ we have $\left(\chi^{2}=\right.$ 95.4): $\Omega_{d, 0}=0.2, \Omega_{\lambda, 0}=-0.1, \Omega_{\mathcal{U}}=0.2, \Omega_{\Lambda_{(4)}, 0}=0.7$.

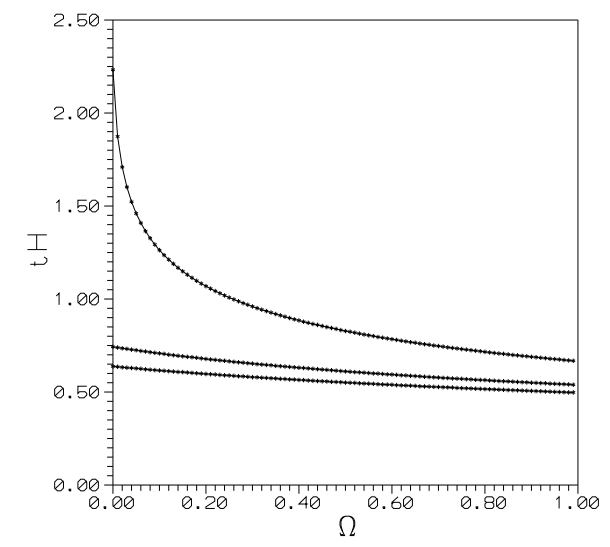

FIG. 3: The age of the universe $t_{0}$ in units of $H_{0}^{-1}$ for the brane models with dust $\left(0 \leq \Omega_{m, 0} \leq 1\right.$ on the horizontal axis). Here $\Omega_{\mathcal{U}, 0}=\Omega_{k, 0}=0, \Omega_{\lambda, 0}=0,0.05,0.1$ (top, middle, bottom).

Now let us briefly discuss the effect of brane parameters and dark energy onto the age of the universe which 
according to (11) is given by

$$
\begin{array}{r}
H_{0} t_{0}=\int_{0}^{1}\left\{\Omega_{G R, 0} x^{-3 \gamma+4}+\Omega_{\lambda, 0} x^{-6 \gamma+4}\right. \\
\left.+\Omega_{\mathcal{U}, 0}+\Omega_{k, 0} x^{2}+\Omega_{\Lambda_{(4)}, 0} x^{4}\right\}^{-\frac{1}{2}} x d x,
\end{array}
$$

where $x=a / a_{0}$. We made a plot for the dust $\gamma=1$ on the brane in Fig. 3 which shows that the effect of quadratic in energy density term represented by $\Omega_{\lambda}$ is to lower significantly the age of the universe. The problem can be avoided, if we accept the dark energy $\gamma=-1 / 3$ [5] on the brane, since the dark energy has a very strong influence to increase the age [16]. In Fig. 14we made a plot for this case which shows how the dark energy enlarges the age.

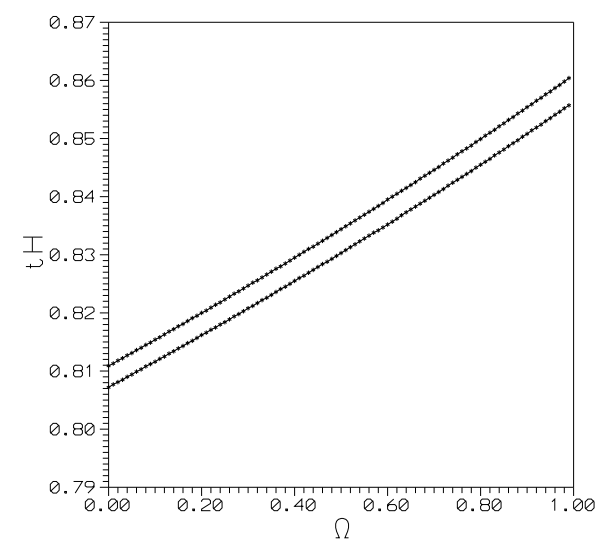

FIG. 4: The age of the universe $t_{0}$ in units of $H_{0}^{-1}$ for the brane models with dark energy on the brane $\left(0 \leq \Omega_{d, 0} \leq 1\right.$ on the horizontal axis). Here $\Omega_{\mathcal{U}, 0}=0.2, \Omega_{\lambda, 0}=0.05,0$ (top, bottom) which shows weaker influence of the brane effects to increase the age.

Finally, let us study the angular diameter test for brane universes. The angular diameter of a galaxy is defined by

$$
\theta=\frac{d(z+1)^{2}}{d_{L}}
$$

where $d$ is a linear size of the galaxy. In a flat dust $(\gamma=1)$ universe $\theta$ has the minimum value $z_{\min }=5 / 4$. It is particu arly interesting to notice that for flat brane models with $\Omega_{\lambda} \approx 0, \Omega_{\Lambda_{(4)}} \approx 0$ the dark radiation can enlarge the minimum value of $\theta$ while the ordinary radiation lowers this value [10], i.e.,

$$
z_{\min }=\frac{1}{2 \mathcal{U}}\left(\Omega_{\mathcal{U}}-1+\sqrt{3 \Omega_{\mathcal{U}}+1}\right) \geq \frac{5}{4}
$$

for $\Omega_{\mathcal{U}} \leq 0$. This is a general influence of negative dark radiation onto the angular diameter size for brane models. One can also notice that there exists a restriction on the amount of negative dark radiation coming from (13) $\left(\Omega_{\mathcal{U}} \geq-1 / 3\right)$ which can serve as a test for the admissible

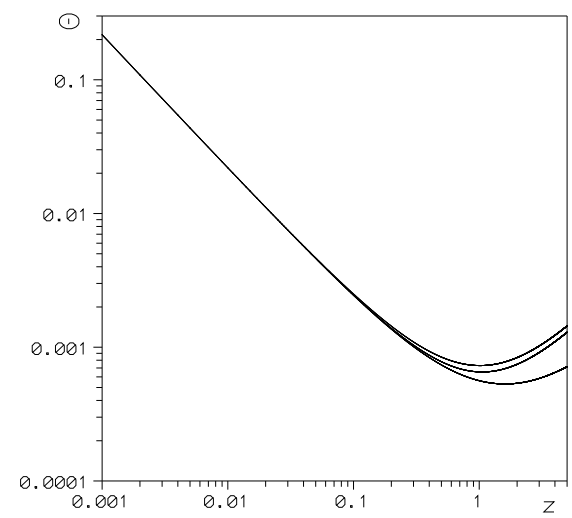

FIG. 5: The angular diameter $\theta$ for $\Omega_{\lambda}=0.1, \Omega_{m}=$ $0.3, \Omega_{\Lambda_{(4)}}=0.72$, and the two values of $\Omega_{\mathcal{U}}=0.1,-0.1$ (top, middle) in comparison with the model of Ref. [3] with $\Omega_{m}=0.28, \Omega_{\Lambda_{(4)}}=0.72$ (bottom).

value of $\Omega_{\mathcal{U}}=-1 / 3\left(z_{\min }=2\right)$ in order to observe the minimum.

More detailed analytic and numerical studies [16] show that the increase of $z_{\text {min }}$ is even more sensitive to negative values of $\Omega_{\lambda}\left(\varrho^{2}\right.$ contribution $)$. Similarly as for the dark radiation $\Omega_{\mathcal{U}}$, the minimum disappears for some large negative $\Omega_{\lambda}$. Positive $\Omega_{\mathcal{U}}$ and $\Omega_{\lambda}$ make $z_{\text {min }}$ decrease. In Fig. 5 we present a plot from which one can see the sensitivity of $z_{\text {min }}$ to $\Omega_{\mathcal{U}}$. We have also checked [16] that the dark energy $\Omega_{d}$ has very little influence onto the value of $z_{\text {min }}$.

* Electronic address: mpdabfz@uoo.univ.szczecin.pl

$\dagger$ Electronic address: godlows@oa.uj.edu.p

¥ Electronic address: uoszydlo@cyf-kr.edu.p

[1] P. Hořava and E. Witten, Nucl. Phys. B460 (1996), 506; ibid B475, 94.

[2] L. Randall and R. Sundrum, Phys. Rev. Lett., 83 (1999), 3370; ibid 83 (1999), 4690.

[3] S. Perlmutter et al., Ap. J. 517, (1999) 565.

[4] A. G. Riess et al. Astron. J. 116 (1998) 1009.

[5] R.R. Caldwell, astro-ph/9908168, S. Hannestad and E. Mörstell, astro-ph/0205096, P.H. Frampton, astro$\mathrm{ph} / 0209037$.

[6] A.G. Riess et al., Ap. J. 560 (2001), 49.

[7] B.P. Schmidt et al. - private communication.

[8] M. Szydłowski, M.P. Dąbrowski. and A. Krawiec, Phys. Rev. D66 (2002), 0640XX (hep-th/0201066).

[9] M.P. Dąbrowski, Ann. Phys (N. Y.) 248 (1996) 199.

[10] M.P. Dạbrowski and J. Stelmach, Astron. Journ. 92 (1986), 1272; ibid 93 (1987), 1373.

[11] M.P. Da̧browski and J. Stelmach, Astron. Journ. 97 (1989), 978.

[12] A. Campos and C.F. Sopuerta, Phys. Rev. D63, 104012 (2001).

[13] G. Efstathiou, Mon. Not. R. Astr. Soc. 303 (1999), 147.

[14] Vishwakarma, Gen. Rel. Grav. 33 (2001), 1973. 
[15] P. Singh, R.G. Vishwakarma and N. Dadhich, hep- preparation. th/0206193.

[16] M.P. Dąbrowski, W. Godłowski, and M. Szydłowski, in 